\title{
Two-step phase shifting for optical nondestructive testing
}

\author{
Xiang-Feng Meng, Xiang Peng, Lu-Zhong Cai, Yu-Rong Wang, \\ and Zhi Gao
}

A novel method simplifies the process of interferometric computation while increasing speed and decreasing storage requirements.

With the rapid development of high-quality CCDs and fast digital-image processing, phase-shifting interferometry (PSI) has emerged as a powerful means of optical noncontact and nondestructive testing. Combined with techniques such as holographic microscopy and 3D metrology, PSI is particularly well suited to evaluate wafers and optoelectronic devices. Classical interferometry provides information about an object by recording the fringe patterns generated by the interference of two illuminating laser beams. In PSI, a single laser beam is split into a 'reference' beam and an 'object' beam that are later recombined. Changing the phase angle of the reference beam stepwise during testing gives additional information that can be analyzed using appropriate algorithms. The attraction of PSI is that it is very accurate and precise. A disadvantage is that it requires at least three phase-shifted interferograms, which increases computing time and data storage requirements. Alternative approaches that have been tried include subtraction operations and a 'Laplacian differentiation' algorithm, but neither is optimal.

To simplify the recording and computation process, we have introduced a two-step algorithm in PSI for an arbitrary phase shift $\delta(0<\delta<\pi)$, ${ }^{1,2}$ in which two interferograms are required to retrieve the wavefront of the object being tested. In addition, it is necessary either to directly characterize the object and reference wave intensities, ${ }^{2}$ or to assess the reference wave intensity and solve a related equation. ${ }^{1}$ Figure 1 shows the experimental setup for phase-shifting digital holography. ${ }^{2}$ Light is redirected by a mirror, $\mathrm{M}_{1}$, and then split, $\mathrm{BS}_{1}$, into an object beam and a reference beam. The former is redirected by mirror $\mathrm{M}_{2}$, focused by lens $\mathrm{L}_{01}$, spatially filtered by a pinhole, $\mathrm{PH}_{1}$, and then collimated by lens $\mathrm{L}_{1}$. The object Obj (a so-called Newport resolution target) is placed in the plane behind $\mathrm{L}_{1}$. Similarly, the reference beam is reflected by a mirror attached to a

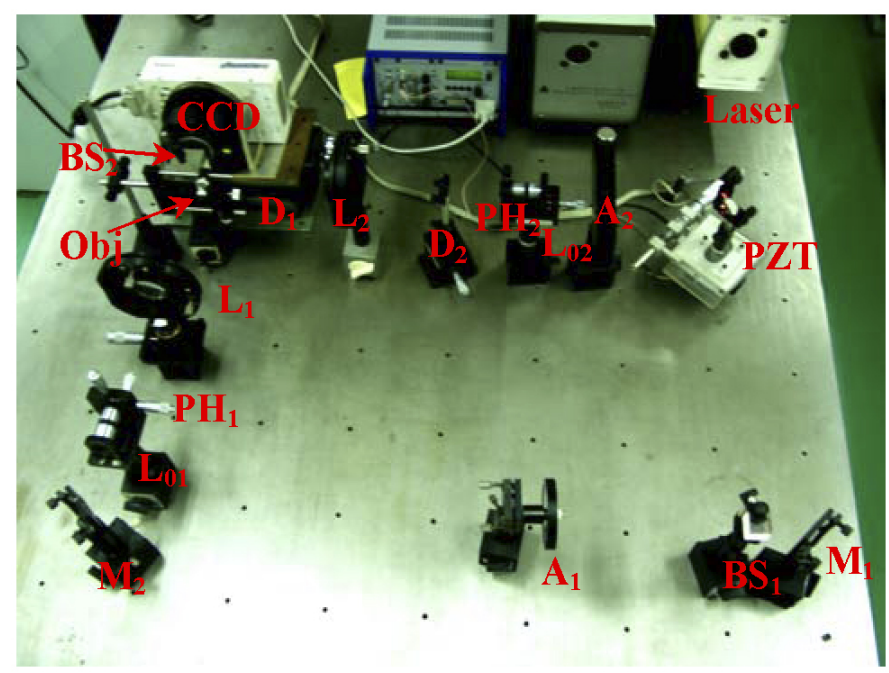

Figure 1. The experimental setup for phase-shifting digital holography. A: Attenuator. BS: Beam splitter. D: Aperture. L: Lens. M: Mirror. Obj: Object. PH: Pinhole. PZT: Piezoelectric transducer.

piezoelectric transducer (Physik Instrumente, Karlsruhe, Germany) controlled by a computer, $\mathrm{PC}_{1}$. It is focused by a lens, $\mathrm{L}_{02}$, filtered by a pinhole, $\mathrm{PH}_{2}$, collimated by a lens, $\mathrm{L}_{2}$, and finally redirected by beam splitter, $\mathrm{BS}_{2}$, to propagate to a recording CCD camera $(1024 \times 1536$ pixels with pixel pitch of $9 \mu \mathrm{m}$ : Photometrics, Tucson, AZ), where the two waves come together, forming interferograms. $A_{1}$ and $A_{2}$ are two attenuators used to modulate the light intensity, and $D_{1}$ and $D_{2}$ are two apertures. The phase shift of the reference wave is realized by moving the PZT. Figure 2(a) and (b) shows the recorded interferograms $I_{1}$ and $I_{2}$. The object wave intensity $I_{0}$ and reference wave intensity $I_{r}$ are given in Figure 2(c) and (d). The reconstructed object image in its original plane (central portion, red box) is displayed in Figure 3(a), and extracted and enlarged as Figure 3(b). The retrieved resolution is as high as $8.77 \mu \mathrm{m}$ (group 5, number 6), a result that verifies the effectiveness of the proposed two-step PSI. ${ }^{2}$ 


\section{Newsroom}

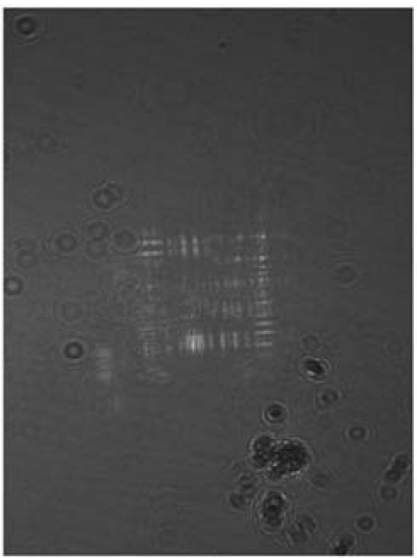

(a)

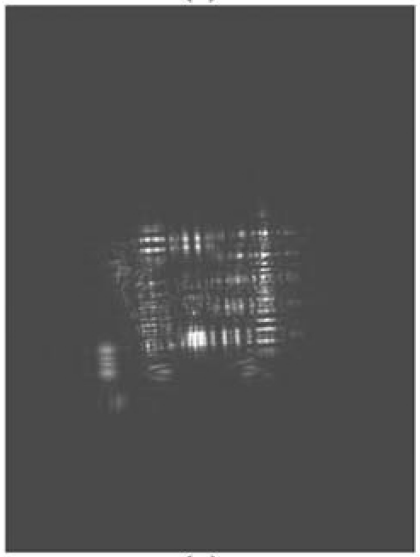

(c)

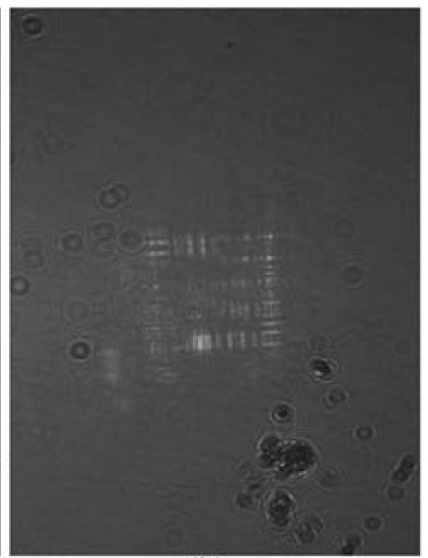

(b)

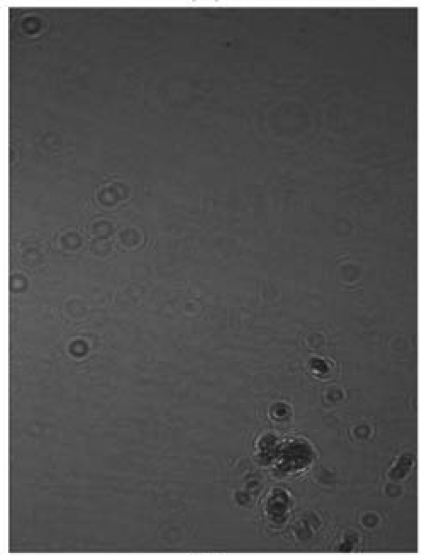

(d)

Figure 2. $(a, b)$ Recorded intensity maps of $I_{1}$ and $I_{2}$, respectively, (c) the object wave intensity $I_{0}$, and (d) the reference wave intensity $I_{r}$.

To further decrease the storage burden and avoid extra measurements, we recently modified the two-step PSI algorithm and applied it to a sinusoidal fringe projection system. ${ }^{3}$ This approach removes the direct current (DC) component, leaving only two DC-term-suppressed fringe patterns. The DC terms in the two phase-shifted patterns can be extracted and suppressed using either an averaging technique in the spatial domain or a low-pass filtering operation in the Fourier frequency domain.

We carried out an experimental measurement of a complex object surface using a homemade, fringe projection-based $3 \mathrm{D}$ system. ${ }^{3}$ Two $\pi / 2$-phase-shifted sinusoidal fringes generated by a computer were loaded into a suitable device (Mitsubishi, MD-150S) and projected onto the surface of a face mask. A CCD camera (Daheng, DH-HV1303UM, $1280 \times 1024)$ was used to capture the two reflected fringe patterns $I_{1}$ and $I_{2}$. A $2 \mathrm{D}$ $80 \times 480$ Hanning filter was used to extract the DC term. Following inverse Fourier transform and a subtraction operation,

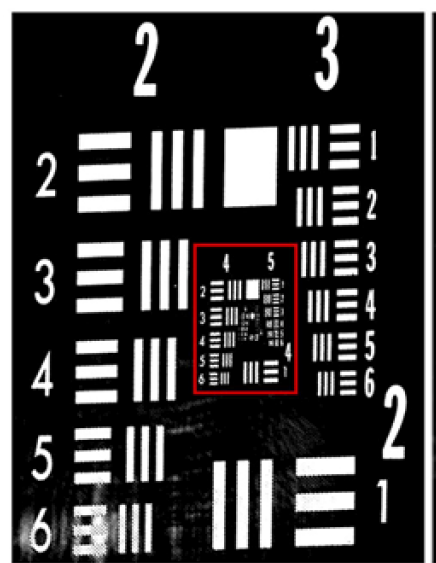

(a)

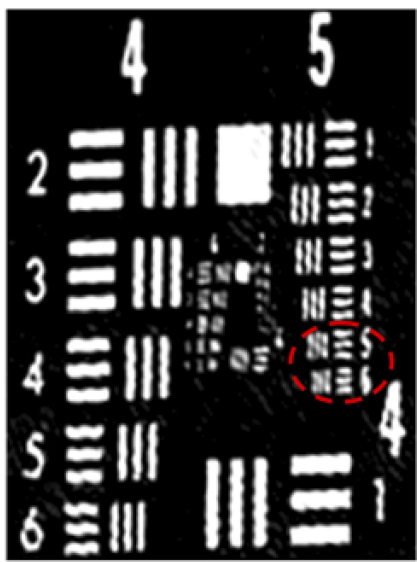

(b)
Figure 3. Optical experimental results using a resolution target as input image: (a) reconstructed image; (b) enlarged central portion of (a).
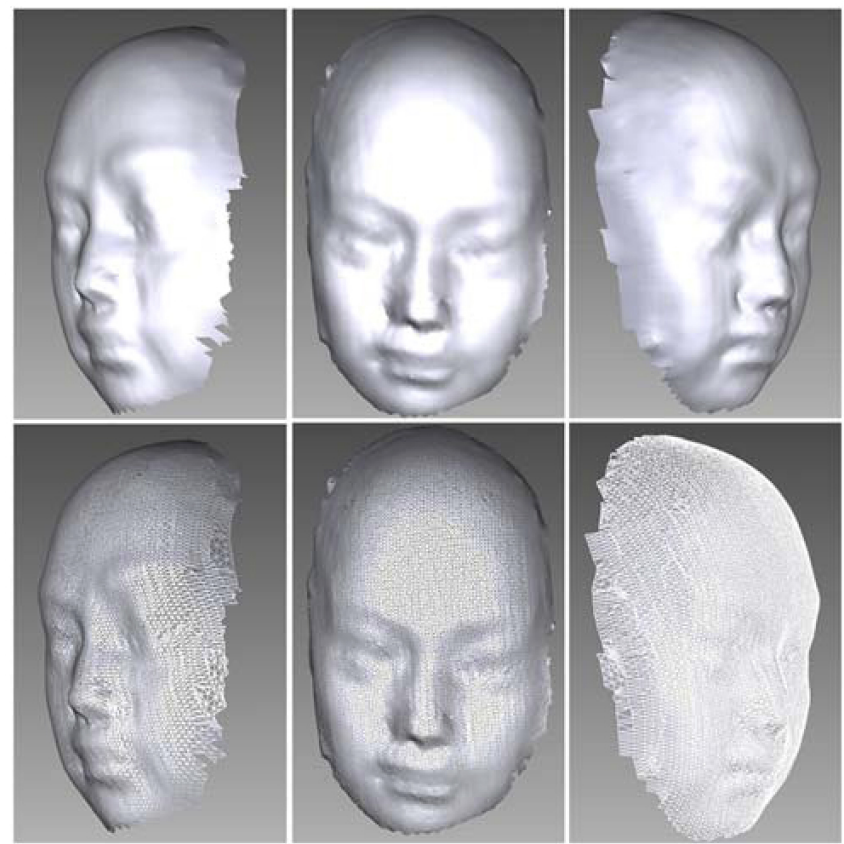

Figure 4. Experimental results using the modified two-step phaseshifting approach. (top row) Typical 3D geometries of a human-face mask in shaded mode. (bottom row) The corresponding $3 D$ geometries in wireframe mode.

two DC-term-suppressed intensities, $I_{1 s}$ and $I_{2 s}$, were obtained. Figure 4 shows the recovered 3D geometry in shaded mode (top row) and wireframe mode (bottom row) following wavefront

Continued on next page 
retrieval, phase extraction, phase unwrapping, system calibration, Gaussian smoothing, and noise reduction. The experimental system produces satisfactory results using the modified two-step phase-shifting approach, and the 3D shape is easily acquired. ${ }^{3}$

In conclusion, we have described a two-step PSI technique and verified it for digital holography. To further decrease the storage burden and avoid extra measurements, we modified the initial approach to require only two DC-term-suppressed phase-shifted intensities to realize wavefront reconstruction. We experimentally measured a complex object surface using a home-made fringe projection-based 3D system, with satisfactory results. The advantages of this method lie in its simplicity, increase in processing speed, and decreased storage burden. The technique opens up numerous applications in the field of optical nondestructive testing. Our next steps will focus on fringe parallel-processing techniques and a color fringe projection system to enable high-speed, real-time 3D shape measurement in, for example, medical imaging, feature films, and games.

This work is supported by the National Natural Science Foundation of China (grants 60775021 and 60777008), the Science and Technology Bureau of Shenzhen (grant 200734), and a China postdoctoral Science Foundation-funded project.

\section{Author Information}

\section{Xiang-Feng Meng and Xiang Peng \\ College of Optoelectronic Engineering \\ Shenzhen University \\ Shenzhen, China}

Xiang-Feng Meng obtained his PhD in 2008 from Shandong University with a specialty in optical engineering. Currently he works as a postdoctoral fellow in Shenzhen University. His research interests include optical nondestructive testing, phaseshifting interferometry, and optical information security. He has coauthored more than 30 professional papers in international publications.

Xiang Peng has research experience in optical science and engineering. He has authored and co-authored over 100 technical papers in international publications. He also holds seven patents in the fields of 3D imaging and modeling, and optical cryptography. His honors include a research fellowship from the Alexander von Humboldt Foundation in Germany and the Science and Technology Advancement Award of the Chinese Education Ministry in 1992.

\author{
Lu-Zhong Cai and Yu-Rong Wang \\ Department of Optics \\ Shandong University \\ Jinan, China
}

\section{Zhi Gao}

Department of Biomedical Engineering

Clemson University

Clemson, SC

References

1. X. F. Meng, L. Z. Cai, X. F. Xu, X. L. Yang, X. X. Shen, G. Y. Dong, and Y. R. Wang, Two-step phase-shifting interferometry and its application in image encryption, Opt. Lett. 31 (10), pp. 1414-1416, 2006. doi:10.1364/OL.31.001414

2. X. F. Meng, L. Z. Cai, Y. R. Wang, X. L. Yang, X. F. Xu, G. Y. Dong, $X$. X. Shen, and X. C. Cheng, Wavefront reconstruction by two-step general ized phase-shifting interferometry, Opt. Commun. 281 (23), pp. 5701-5705, 2008. doi:10.1016/j.optcom.2008.08.010

3. X. F. Meng, X. Peng, L. Z. Cai, A. M. Li, J. P. Guo, and Y. R. Wang, Wavefront reconstruction and three-dimensional shape measurement by two-step DC-term-suppressed intensities, Opt. Lett. 34 (8), pp. 1210-1212, 2009. 